\title{
BIOLOGÍA Y CRÍA DE LA MOSCA DEL ESTABLO Stomoxys calcitrans $\mathrm{L}$.
}

Cristina Vargas-Chacón ${ }^{1}$, Arturo Solórzano Arroyo ${ }^{1}$

\section{RESUMEN}

Biología y cría de la mosca del establo Stomoxys calcitrans L. El estudio se realizó en el Laboratorio de Servicios de Fitoprotección del INTA, San José, Costa Rica. Se estudiaron elementos de la biología del insecto que contribuyeron a reproducir una colonia de la mosca del establo hasta su domesticación después de cinco generaciones, a partir de una población silvestre capturada en rastrojos de piña en San Carlos de la provincia de Alajuela. El estudio permitió conocer elementos básicos de su biología, que contribuyeron a la reproducción de los estadios de huevo, larva, pupa y adulto en condiciones de laboratorio. A una temperatura entre $26-28{ }^{\circ} \mathrm{C}$ y una humedad relativa entre $65-75 \%$, se logró un ciclo biológico de 18 días, el cual se alargó hasta 43 días cuando las condiciones nutricionales fueron insuficientes, manteniéndose el insecto en estadio larval. Los adultos se mantuvieron vivos hasta por 28 días. Cabe destacar que se requirió dotar a las moscas silvestres de una solución azucarada de leche en polvo al $10 \%$, además de sangre con anticoagulante para que se iniciara la oviposición; luego de cuatro ciclos la leche no fue necesaria. Además se elaboró el protocolo de cría que garantiza la cantidad de individuos necesarios para futuros estudios. La mosca del establo afecta directamente la ganadería e indirectamente la sostenibilidad de las explotaciones agrícolas que dejan en el campo grandes cantidades de rastrojos en proceso de descomposición, que aunado a un mal manejo beneficia la propagación de este tipo de díptero.

Palabras clave: Dípteros, dieta, ciclo biológico, hematófagos.

\section{INTRODUCCIÓN}

La mosca del establo es una plaga hematófaga que afecta las explotaciones pecuarias a nivel mundial (Taylor y Berkebile 2006) y nuestro país no es la excepción, ya que desde el año 1987 se han reportado daños económicos en ganadería debidos a esta plaga (Herrera et al. 1994). El macho y la hembra succionan sangre en varias ocasiones para completar su desarrollo y reproducción. Sin la sangre no se completa la formación de huevecillos (Foil y Hogsette 1994, Zumpt 1973, Salem 2012 y Justin 2008), los cuales se ovipositan sobre materia orgánica en proceso de descomposición (residuos de cosecha), abonos orgánicos de origen animal no tratados como cerdaza, gallinaza y pollinaza que permiten que el insecto cumpla su ciclo reproductivo (Martínez y Lumaret 2006). Estos insectos succionadores de sangre, causan dolorosas

1 Instituto Nacional de Innovación y Transferencia en Tecnología Agropecuaria, INTA. Costa Rica. cvargas@inta.go.cr, asolorzano@inta.go.cr. Sede del Laboratorio de Servicios de Fitoprotección del INTA. Sabana Sur, San José. 
picaduras a los animales que afectan la ganancia de peso y producción de leche, afectando también la vitalidad del animal (Harwood y James 1979, Campbell et al. 2001 y Gilles et al. 2008). Consecuentemente los animales se agrupan, aumentando el estrés por calor y pérdida de peso (Foil y Hogsette 1994).

Para implementar mecanismos de control de la plaga es indispensable conocer la biología de la mosca y disponer del material biológico necesario para implementar estudios que soporten una propuesta para el manejo integrado de la mosca del establo. Establecer una colonia de moscas del establo es un aspecto fundamental para que se inicien las investigaciones relacionadas con el control químico y biológico de la plaga (Singh y Moore 1985). La colonia puede establecerse a partir de moscas de campo, sin embargo, el tiempo requerido para que se adapten al laboratorio puede ser largo, ya que las moscas recién colonizadas se resisten a alimentarse y a aparearse (Singh y Moore 1985 y Rodríguez 2007).

Desde el año 1924 se reportan técnicas para la cría de larvas de Stomoxys calcitrans, dichas técnicas van desde la crianza utilizando heces de animales, medios sintéticos, subproductos vegetales como fuente de carbohidratos y proteínas (Parr 1959, Bakri 1959, Zumpt 1973 y Hargett y Goulding 1962). También se han reproducido larvas en jarras plásticas de 4,5 ml en un medio de cultivo CSMA compuesto por salvado de alfalfa, levadura seca, colesterol, malta y agua (Ashrafi 1964). Larvas de otras especies de moscas hematófagas fueron criadas por Schmidt et al. (1967 y 1968) utilizando una mezcla de pulpa de caña de azúcar molida, harina de trigo, plasma bovino deshidratado, bicarbonato de sodio y agua. Las dietas a base de subproductos de la caña de azúcar han sido muy exitosas en Brasil. Se logró establecer eficientemente una colonia haciendo varias modificaciones tanto en las cajas de cría como en la dieta de alimentación de los inmaduros (Rodríguez 2007). Se evaluaron siete sustratos para el desarrollo larval incluyendo hojas y tallos de caña de azúcar, distintas especies de pastos forrajeros (zacate elefante, ensilaje de pasto kikuyo, pasto Rhodes y estiércol maduro), conforme se presentaron altos niveles de celulosa se redujo el tiempo de reproducción y a niveles de $\mathrm{pH}$ ligeramente ácido se aumentó el porcentaje de sobrevivencia de huevo a adulto (Gilles et al. 2008).

Otros materiales que han sido utilizados en las dietas, con el propósito de disminuir la compactación del medio de cultivo, son las cáscara de avena, virutas de madera, vermiculita, alimento para perros, levadura de cerveza, paja y cáscaras de maní entre otros (Doty 1937, Campau et al. 1953, Champlain et al. 1954, Berkebile et al. 2009 y Salem et al. 2012). Para la alimentación de los adultos se describe el uso de sangre bovina con anticoagulante (Hargett y Gouding 1962 y Schmidt et al. 1967, 1968).

\section{MATERIALES Y MÉTODOS}

El estudio se realizó en el Laboratorio de Servicios de Fitoprotección del Instituto Nacional de Innovación y Transferencia en Tecnología Agropecuaria (INTA), ubicado en la provincia de San José, Cantón Central, distrito Mata Redonda. ( $\left.9^{\circ} 55^{\prime} 59.21^{\prime \prime} \mathrm{N} 84^{\circ} 7^{\prime} 7.466^{\circ} \mathrm{O}\right)$. La cría se realizó en un cuarto a una temperatura entre $26-28^{\circ} \mathrm{C}$, a una humedad relativa entre $65-75 \%$ y un fotoperiodo de 12:12 horas luz:oscuridad. Para mantener la temperatura y humedad relativamente controladas se colocó en el área un calentador portátil de cerámica Optimus H 7004 1000W y un humidificador tipo consola de 12 galones, Air King Modelo H12400.

Las moscas con las que se iniciaron los estudios, denominadas silvestres (Fs) fueron capturadas en campo, en la zona norte del país, específicamente en Pital de San Carlos (provincia de Alajuela), mediante colocación de trampas de 
tela azul/negra de cono invertido (Vavoua) y triangular (NZI) (Guilles et al. 2007) para moscas en fincas piñeras una semana después de la derriba en verde (corte de la planta sin usar desecantes) con trituradora Seppi M Midiforst dt 150; además, se capturaron las que emergían de rastrojos de piña húmedos y frescos llevados al laboratorio y colocados en cajas de madera. Con la ayuda de aspiradores con boquilla ancha, se pasaron las moscas a cajas limpias de vidrio, madera y plástico de diferentes tamaños y con malla antiáfidos para facilitar la aireación y evitar la entrada de insectos contaminantes y se colocaron en el cuarto de cría.

Para la alimentación se utilizó sangre bovina con anticoagulante, ya que en pruebas preliminares, las moscas no se alimentaron de sangre sin anticoagulante ni de órganos con alto contenido sanguíneo como hígado de res y pollo. Se utilizaron bolsas comerciales para la extracción de sangre CPDA- 1 con citrato de sodio como anticoagulante $(2,63 \mathrm{~g} / 100 \mathrm{ml})$. La sangre extraída fue fraccionada en tubos estériles, con aproximadamente $15 \mathrm{ml}$ de sangre por tubo y almacenada en refrigeración a $5{ }^{\circ} \mathrm{C}$. Las moscas se alimentaron diariamente por las mañanas para ello se utilizó una esponja embebida con la sangre tibia $\left(37^{\circ} \mathrm{C}\right)$, que se colgó dentro de la caja de cría, para lo cual se utilizó un clip de metal como gancho.

\section{Sexado de adultos}

El sexado de adultos se realizó en moscas muertas mediante la separación y forma de los ojos, lo cual permitió medir la cantidad de machos y hembras Fs provenientes de campo y de las obtenidas en la cría. Además se midió el índice frontal (IF) y la longitud del cuerpo del insecto diferenciado entre machos y hembras. El índice frontal (IF), es la relación entre la distancia EO (espacio frontal del vertex) entre LO (longitud del ojo) (Masmeatathip et al. 2006).

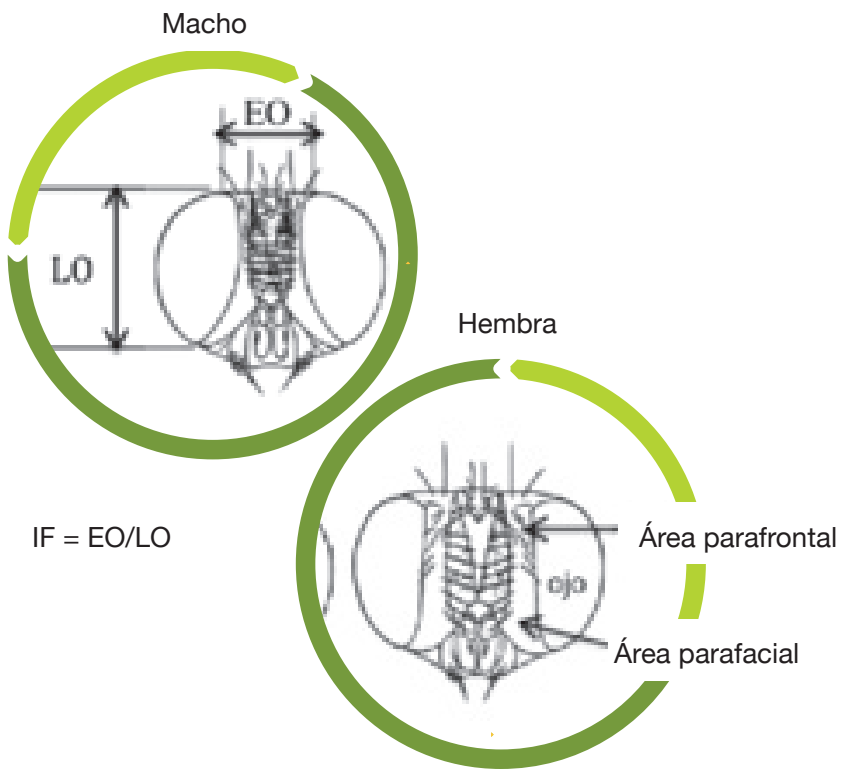

Figura 1. Macho y hembra. Detalle en la separación y forma de los ojos, área parafrontal y área parafacial. San José, Costa Rica, 2013.

Fuente: Salem 2012, adaptado de Zumpt 1973. Modificado por Vargas 2013.

\section{Recolecta de huevos}

En las cajas de cría con los adultos, se colocó un material que sirviera de atrayente para que las moscas grávidas ovipositaran; para ello se probaron dos sistemas: uno colocando trozos de pinzote de plátano semidescompuesto y otro donde se colocó en una caja petri con la dieta para larvas. Diariamente se puso material nuevo y se observó el del día anterior al estereoscopio para verificar y determinar el día de la oviposición. El material junto con los huevos fue trasladado a las cajas de cría de larvas.

\section{Desarrollo de larvas y pupas}

Para el desarrollo de las larvas se evaluó pinzote de plátano picado en trozos de aproximadamente $5 \mathrm{~cm}$, en proceso de descomposición y una dieta compuesta de salvado de trigo (312 g), harina de pescado $(75 \mathrm{~g})$ aserrín de madera $(125 \mathrm{~g})$ en agua destilada (1000 ml) (Berkebile y 
Taylor 2013) ${ }^{2}$. Los huevos recolectados, se colocaron sobre el material y se colocó una toalla de papel húmeda a fin de mantener la humedad (Singh y Moore 1985).

Se utilizaron cajas plásticas de $708 \mathrm{~mm}^{3}$ de volumen $(15 \times 8 \times 5 \mathrm{~cm})$, marca Ziploc con tapa plástica a la cual se le realizó un agujero y se le pegó malla antiáfidos para facilitar la aireación e impedir el ingreso de otros insectos al sistema. Dentro de las cajas se colocaron cada uno de los materiales utilizados para alimentación de las larvas, cubriendo el fondo con una capa de $3 \mathrm{~cm}$ del material. Para la recolecta de pupas se siguió el procedimiento planteado por Berkebile et al. (2009), donde se colocó en la caja de cría de larvas, una esponja humedecida con agua y envuelta con una tela negra hasta la formación de las pupas.

Las pupas provenientes de la Fs, se trasladaron a las cajas de cría de adultos, donde se evaluó la emergencia, desarrollo y comportamiento de adultos y oviposición. En cuanto fueron muriendo se sexaron y se midió la longitud de su cuerpo y el índice frontal. Las siguientes generaciones fueron denominadas F1, F2 y así consecutivamente. Se llevó un registro de la fecha en que se colocaron los huevos en las bandejas con el pinzote, humedad relativa, la temperatura, fechas de eclosión de larvas, días de los estadios larvales, fecha de pupación y fecha de emergencia de adultos.

\section{RESULTADOS Y DISCUSIÓN}

\section{Población inicial}

Las moscas adultas recolectadas en campo fueron trasladadas lo más pronto posible al laboratorio; sin embargo, durante el transporte muchas de ellas murieron, llegando vivas menos del $50 \%$ de los adultos capturados. La población de moscas adultas obtenidas de rastrojos una semana después de la derriba en verde, fue más efectiva, ya que las larvas se mantuvieron en el rastrojo y el mismo día o al día siguiente de emergencia de los adultos (Fs), fueron trasladados a cajas de cría limpia.

Dado que de los rastrojos salieron varios géneros de dípteros, se verificó la especie previo al traslado de las mismas. Los adultos de la mosca del establo (Figura 2), se reconocieron fácilmente principalmente por su aparato bucal, caracterizado por la presencia de una probosis robusta de color negro, la cual utiliza para perforar la piel y succionar sangre.

2 Berkebile, A; Taylor, D. 2013. Dieta para la cría de la mosca del establo. (Correo electrónico). USDA ARS. University of Nebraska, USA. Comunicación personal.

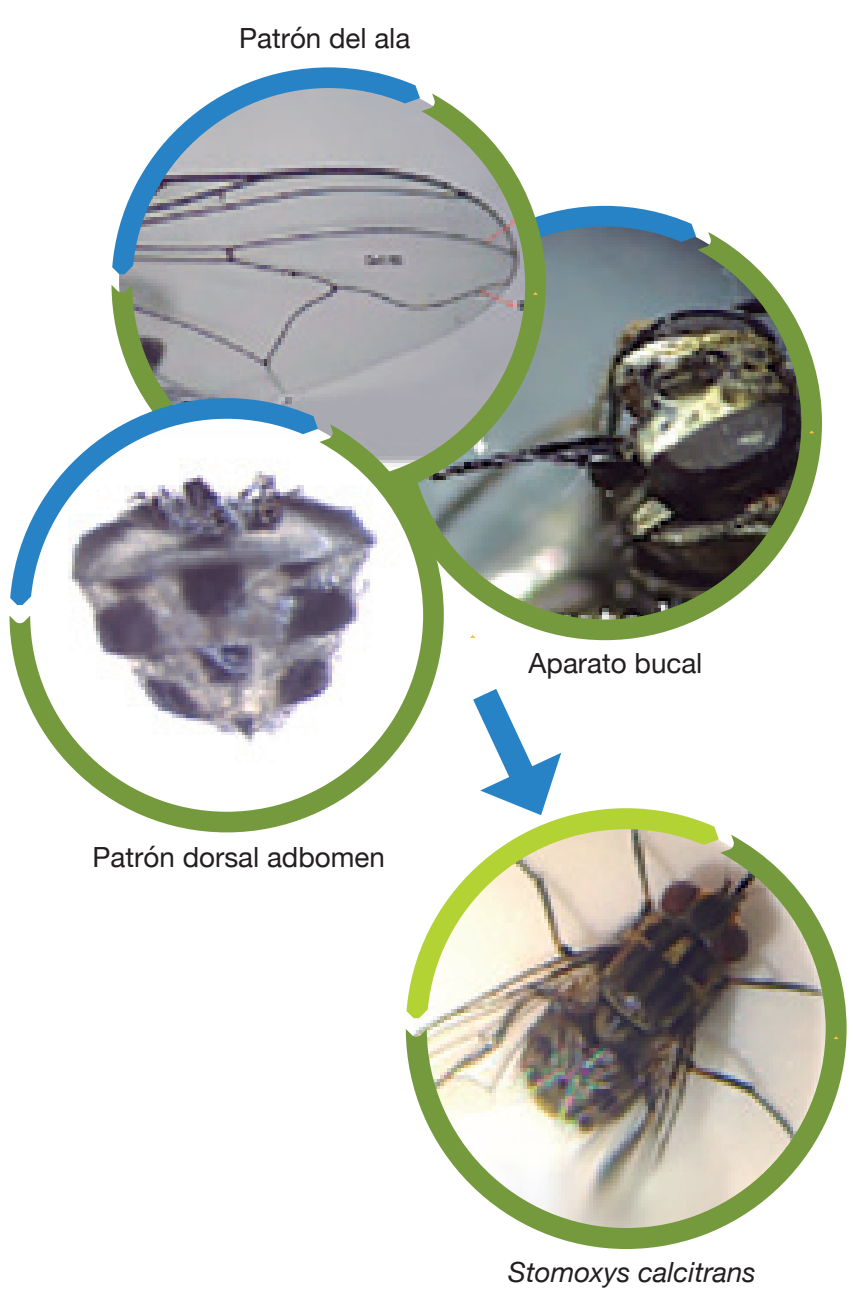

Figura 2. Identificación de adultos de la mosca del establo. San José, Costa Rica, 2013. 
Con una lupa 10X, se pudo observar el patrón de venas del ala, en la cual la vena $\mathrm{M} 1+2$ es ligeramente curveada hacia arriba acercándose, pero nunca tocando la R4+5. En el abdomen de esta mosca (en su porción dorsal) se observó un patrón de manchas oscuras dibujando un tablero de damas. Con este patrón, según Zumpt (1973), se pueden diferenciar especies de Stomoxys. Según la comparación entre patrones abdominales, post mortem, se corroboró que la especie que se manipuló correspondía a la mosca del establo (Figura 3).

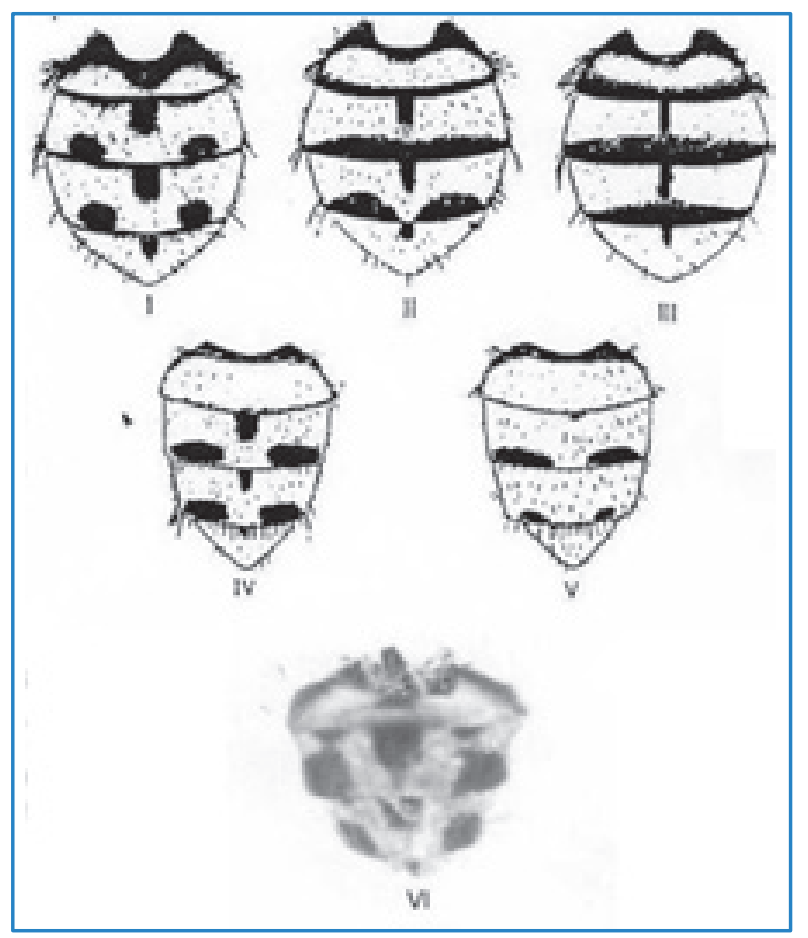

Figura 3. Patrón abdominal de: (I) Stomoxys calcitrans (L.). (II) S. nigra nigra (Mcq.). (III) S. indica (Pic). (IV) S. sitiensis Rond. (V) S. nigra bilineata Grunberg. (Zumpt 1973). (VI) mosca criada en el Laboratorio de Servicios de Fitoprotección del INTA.

\section{Adultos}

\section{Cajas de cría}

Las cajas de vidrio, con malla antiáfidos y marcos de aluminio (Figura 4), fueron consideradas en un inicio una buena opción para la cría de adultos, dado que el material ofrecía una mayor facilidad para la limpieza y por lo tanto una mejor asepsia del sistema de reproducción. Sin embargo, las moscas únicamente se mantuvieron vivas por un período de 24 horas. Se observó que los adultos chocaban constantemente contra el vidrio, por lo que el daño físico por el golpe aceleró su muerte.

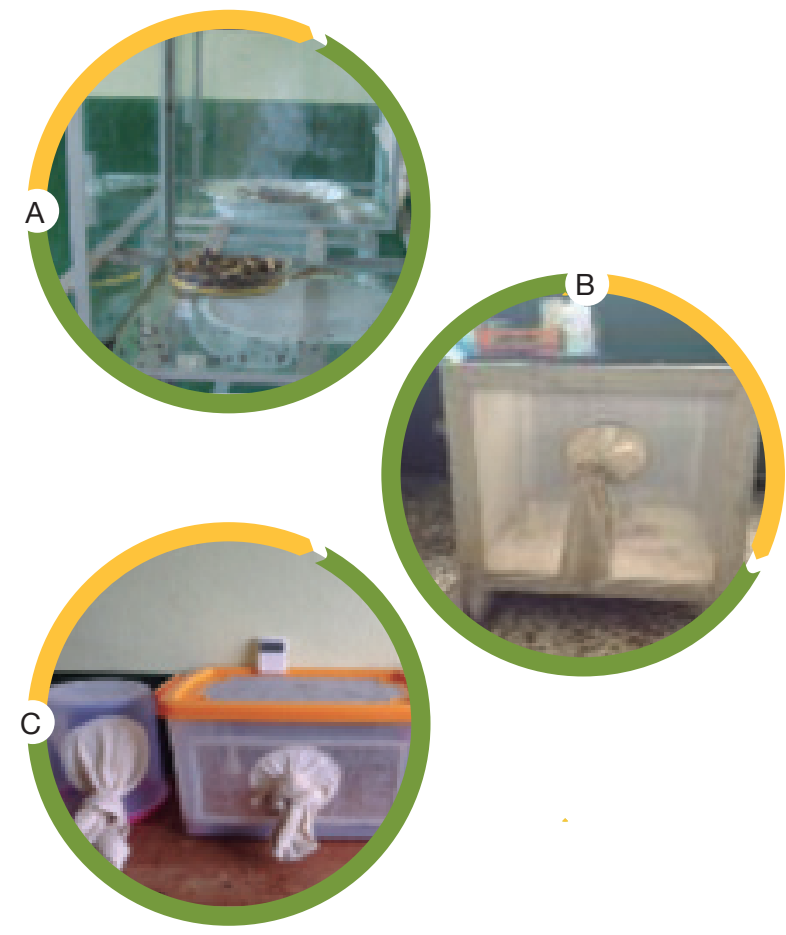

Figura 4. Tipos de cajas probadas para la cría de moscas adultas. A: vidrio, B: madera, C: plástico. San José, Costa Rica, 2013.

En las cajas de madera y plástico (Figura 4B y 4C), las moscas lograron sobrevivir hasta por 28 días, superando en 7 días lo indicado por Singh y Moore (1985). Hay que mencionar, que las cajas de madera por naturaleza son de un material muy poroso, por lo que para obtener un período de vida largo, éstas debieron ser lavadas muy bien con jabón, cloro y agua antes de ser utilizadas a fin de evitar las contaminaciones recurrentes que se produjeron cuando no fueron eficientemente lavadas. Sin el lavado eficiente se presentaron muchos problemas de contaminación del material utilizado para la oviposición, con hongos como Penicillium spp. y Aspergillus spp. El material plástico tiene menos poros que la madera, es más fácil de limpiar y efectivamente se obtuvo una disminución en la contaminación por los hongos mencionados anteriormente. Las cajas plásticas de fondo totalmente plano, resultaron ser las mejores, otras que tenían alguna curvatura o división dejó espacios donde se metieron las moscas quedando atrapadas y causando la muerte temprana de los adultos. 
Tanto en cajas de plástico como en las de madera ingresaron otros dípteros de la familia Drosophilidae, por lo que se debe tener especial cuidado en cerrar todas las aberturas de ingreso de este insecto, a fin de evitar la contaminación de la cría. Se colocó cinta adhesiva alrededor de las puertas de madera y se revisó diariamente la malla antiáfidos. En caso de encontrarse agujeros se taparon con silicona.

\section{Alimentación}

Las moscas silvestres (Fs), se resistieron a alimentarse diariamente obteniéndose una mortalidad del $100 \%$ en un primer intento y de un $80 \%$ en un segundo intento, de ahí que para establecer la colonia fue fundamental no fallar con la colocación de la espuma con sangre, a fin de que el $20 \%$ restante sobreviviera y se iniciara el proceso de adaptación al cautiverio. Una vez que las moscas iniciaron la alimentación con sangre se logró el establecimiento de las siguientes generaciones. El abdomen de las moscas se observó de color rojo y completamente abultado luego de la alimentación (Figura 5).

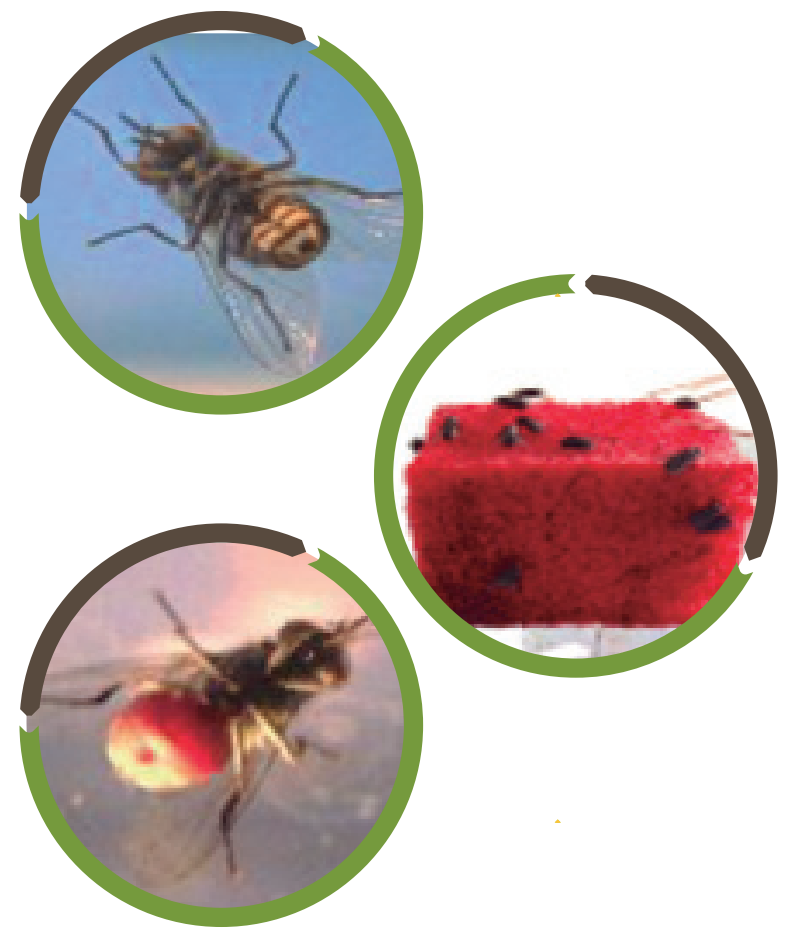

Figura 5. Abdomen de la mosca del establo antes y después de la succión de sangre bovina con anticoagulante. San José, Costa Rica, 2013.

\section{Sexado (machos y hembras Fs)}

El dimorfismo sexual de la mosca del establo facilitó la diferenciación entre machos y hembras, se comprobó que los machos poseen ojos compuestos que se acercan bastante en la parte media frontal sin llegar a ser holópticos y las hembras poseen ojos ampliamente separados (diópticos). El sexado y mediciones realizadas a los adultos Fs, se efectuaron post mortem a partir del grupo de moscas que lograron alimentarse con sangre con anticoagulante.

La mayoría de las moscas adultas capturadas del campo y las llevadas al laboratorio en rastrojo, una semana luego de la derriba en verde (Fs), fueron hembras. Se evaluó una población de 73 moscas de las cuales 40 fueron hembras y 23 machos. Sin embargo en la cría establecida la proporción de machos y hembras llegó a ser de 1:1. Esto podría deberse a que son las hembras las que mayormente llegan a los rastrojos de piña atraídas por un nicho para ovipositar, lo cual es congruente con lo indicado por Solórzano et al. (2013), quienes encontraron que las hembras son las primeras en llegar al rastrojo en descomposición.

\section{Índices frontales y longitud de adultos}

El índice frontal (IF) de las hembras fue mucho mayor que el de los machos. Para la población silvestre (Fs), se obtuvo un IF promedio de 0,56 $\pm 0,04$ para hembras y $0,36 \pm 0,05$ para machos (Cuadro 1). Estos valores no son diferentes de los resultados obtenidos con las poblaciones de moscas de Tailandia donde, Masmeatathip et al. (2006), reportaron valores de 0,55 para hembras y 0,33 para machos.

Las hembras Fs de la mosca del establo, por lo general poseen un cuerpo más largo que el de los machos. Las hembras midieron entre 5 y 7 $\mathrm{mm}$, mientras que los machos entre 4 y $6 \mathrm{~mm}$ de longitud. Ninguna hembra llegó a medir menos de $5 \mathrm{~mm}$ y ningún macho llegó a medir más de $6 \mathrm{~mm}$ de largo (Cuadro 1). 
Cuadro 1. Índices frontales y longitud promedio de la Fs de la mosca del establo, según sexo. San José, Costa Rica, 2013.

\begin{tabular}{|l|c|c|c|c|}
\hline \multicolumn{1}{c|}{ Sexo } & \multicolumn{2}{|c|}{ Hembra $\mathbf{n = 4 0}$} & \multicolumn{2}{c|}{ Macho $\mathbf{n = 2 3}$} \\
\hline \multicolumn{1}{|c|}{ Parámetro } & $\begin{array}{c}\text { Índice } \\
\text { frontal }\end{array}$ & $\begin{array}{c}\text { Longitud, } \\
\mathbf{m m}\end{array}$ & $\begin{array}{c}\text { Índice } \\
\text { frontal }\end{array}$ & $\begin{array}{c}\text { Longitud, } \\
\mathbf{m m}\end{array}$ \\
\hline Valor promedio & 0,558 & 5,82 & 0,359 & 5,50 \\
\hline $\begin{array}{l}\text { Desviación } \\
\text { estándar }\end{array}$ & 0,044 & 0,67 & 0,051 & 0,54 \\
\hline Valor mínimo & 0,390 & 5,00 & 0,279 & 4,00 \\
\hline Valor máximo & 0,640 & 7,00 & 0,461 & 6,00 \\
\hline
\end{tabular}

Estos parámetros podrían ser útiles para evaluar la calidad de la cría conforme se den los ciclos de desarrollo.

\section{Huevos}

\section{Oviposición}

En las cajas de cría con adultos se colocaron unos trozos de pinzote semidescompuesto para estimular la oviposición sobre ese material, se les proporcionó la sangre; sin embargo con el primer grupo de adultos Fs mantenidos en el laboratorio por 28 días no se evidenció ninguna postura. Ya ha sido mencionado por Singh y Moore (1985) y Rodríguez (2007) que el tiempo para establecer una colonia a partir de moscas silvestres se alarga ya que las mismas se resisten a alimentarse y copular. En un segundo intento con otro grupo de Fs se observó que una pequeña cantidad de huevos fueron colocados no sobre el pinzote, sino por toda la caja de cría, incluso se observaron algunos fuera de la misma evidenciando que las hembras sacaban el ovipositor por la malla antiáfidos. Por lo anterior, se recomienda establecer una colonia a partir de pupas provenientes de una colonia establecida, ya que como indica Singh y Moore (1985), se ahorraría tiempo considerable.

Ante la situación expuesta y la sobrevivencia de solo un $20 \%$ de la Fs, se introdujeron constantemente nuevos adultos silvestres a las cajas de cría de adultos y se les proporcionó la solución azucarada con leche en polvo al $10 \%$
(Funes 2013³). A los dos días de esa práctica, se observaron mayores cantidades de huevos. Con este resultado, se cree que la composición de la leche proporcionó alguna (s) sustancia (s) que incidieron en la nutrición y bienestar del insecto, ya que la leche en polvo contiene hidratos de carbono, minerales, proteínas, vitaminas y ácidos grasos (Dos Pinos sf) que pudieron ser necesarios en ese momento. A partir de esta práctica, los adultos se mostraron muy activos e iniciaron la cópula y con ello una mayor oviposición. Cabe mencionar que los trozos de pinzote semidescompuestos utilizados inicialmente, se contaminaban con mucha facilidad y a fin de disminuir los efectos adversos que dichos contaminantes podían tener en el desarrollo del ciclo, se cambió el pinzote por una porción pequeña de la dieta para larvas (salvado de trigo, harina de pescado y aserrín), colocada húmeda en una caja petri dentro de la jaula. Este material colocado en la caja de cría de adultos, únicamente funcionó como soporte para la postura ya que los adultos se alimentan de sangre.

Se requirieron cinco generaciones para obtener producciones significativas de huevos, de ahí que para tener una colonia estable se debe dedicar el tiempo suficiente durante los primeros cinco o seis ciclos de reproducción, luego de los cuales se sincroniza la producción de todos los estadios de la mosca y puede eliminarse el uso de la leche e incluso el calentar la sangre.

\section{Recolección de huevos}

Cuando se observaron huevos sobre la porción de dieta, se pasó el material completo a las cajas de larvas, revisando al final bajo el estereoscopio que no quedaran huevos sin pasar al medio de desarrollo de larvas y si los había, se pasaron con ayuda de un pincel. El traslado de huevos a cajas de cría debe realizarse a más tardar, 24 horas después de ovipositados, de no

3 Funes, C. 2013. Asesoría en Agronomía y Control biológico de plagas (entrevista). San José, Costa Rica. Comunicación personal. 
ser así los huevos se secan mucho y disminuye el porcentaje de eclosión. Una opción a esto es que para los fines de semana se coloque dentro de la caja de adultos una caja de cría para larvas preparada según la metodología, a fin de que la espuma húmeda impida la desecación de los huevos y los lunes solamente se rotule la caja y se pase al área de larvas.

Cada postura de huevos se realizó sobre, entre y debajo del material utilizado para la oviposición, formando grupos de 7 a 15 huevos (Figura 6). Los huevos son de aproximadamente 1 a $2 \mathrm{~mm}$ de largo, alargados en forma de banano y con una sutura longitudinal (Schol 1986, Zumpt 1973, Salem 2012).

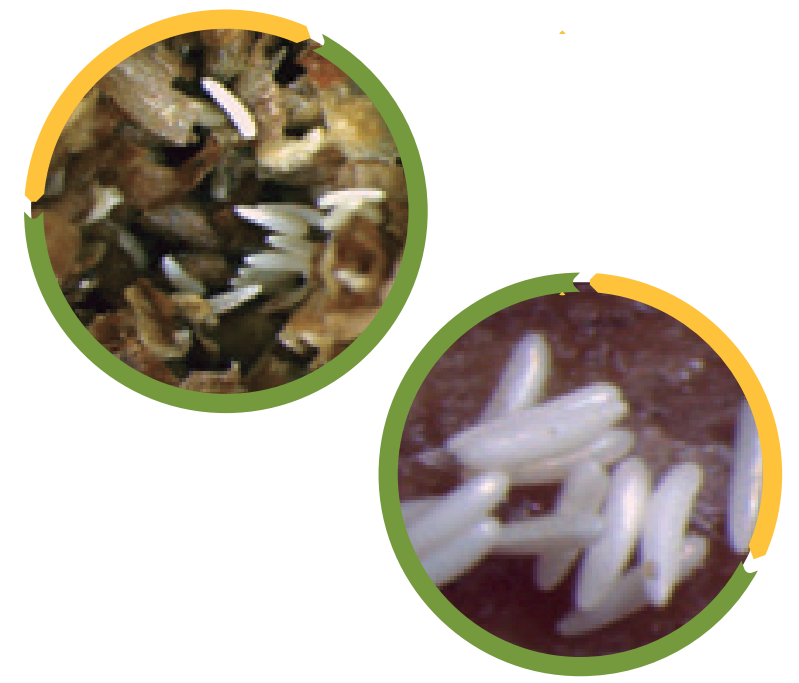

Figura 6. Huevos de Stomoxys calcitrans colocados en grupos. Obsérvese la forma alargada de los mismos. San José, Costa Rica, 2013.

\section{Larvas}

\section{Alimentación y desarrollo de larvas}

Las cajas con huevos fueron puestas dentro de cajas de vidrio, dado que se observó insectos externos queriendo ingresar a la caja de cría. Posteriormente el sistema se cambió y las cajas fueron colocadas sin tapa, dentro de fundas de tela que las protegían de la contaminación y ofrecían una mejor aireación (Figura 7). Con ambas dietas (concentrado de pollo y dieta con salvado de trigo, harina de pescado y aserrín) las larvas eclosionaron entre los cuatro y cinco días luego de ovipositados los huevos en el medio.

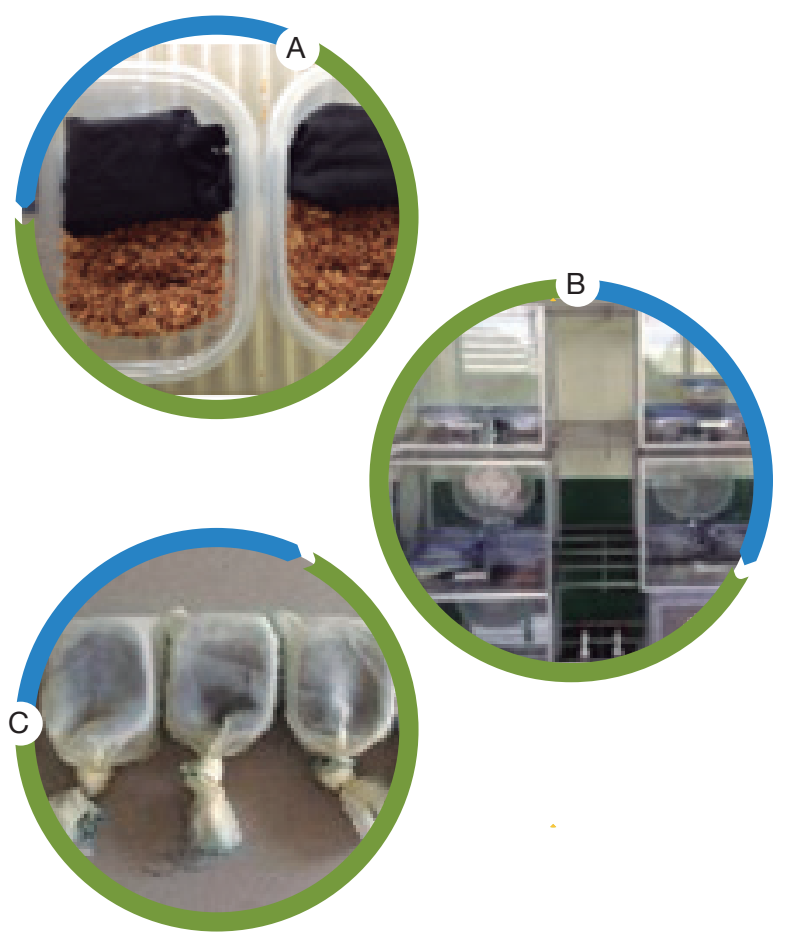

Figura 7. Cajas de cría de larvas. A: Contenido de las cajas. B: Dentro de cajas de vidrio. C: Dentro de fundas de tela. San José, Costa Rica, 2013.

\section{Medio para desarrollo de larvas (pinzote)}

Inicialmente se observó el desarrollo de las larvas sobre pinzote parcialmente descompuesto. Con este, las larvas provenientes de huevos colocados el mismo día emergieron entre los días cuatro y cinco. Sin embargo, el período larval fue muy amplio y no sincronizado. Según los datos la transformación de larvas a pupas duró de 9 a 28 días. Este periodo larval tan largo, extendió el desarrollo del ciclo de cría completo a 52 días (Figura 12). Al parecer, las larvas sobre pinzote pueden permanecer en ese estadio sin pasar a pupa por largo tiempo. Se observaron larvas en este estadio hasta por 40 días, luego de los cuales se descartaron. Los contenidos nutricionales del medio así como la temperatura inciden en la duración de los estadios larvales (Gilles et al. 2005). El estadio larval podría durar cerca de 8 días en condiciones de $26^{\circ} \mathrm{C}$ y $80 \%$ HR (Parr 1962) a varios meses en invierno (Harwood y James 1979), tanto larvas del tercer estadio como pupas podrían extender su período por el frío del inverno (Berkebille et al. 1994). Otros datos indican que la 
eficiencia para llegar a pupa fue de $33 \%$ con una emergencia de adultos del $66 \%$. Los resultados indican que los trozos de pinzote de plátano semidescompuesto, no es un buen material para sincronizar el ciclo de la mosca, quizás porque no reúne los requisitos nutricionales que requiere la larva para pasar a pupa.

\section{Dieta}

Con la dieta, se obtuvieron los menores tiempos de desarrollo del ciclo completo de la mosca, pasando de 52 días utilizando pinzote en el desarrollo de larvas, a 28 días con la dieta. Con este sistema, no se contaron los diferentes estadios por la gran cantidad obtenida.

Cuando se colocaron muchos huevos (+ de 200) en las cajas de cría de larvas, éstas no se desarrollaron de forma homogénea, extendiéndose el periodo de formación de pupas; a pesar de que todos los huevos fueron ovipositados el mismo día unas larvas pasaron a formar pupas al día 10 pero otras se mantuvieron como larvas hasta por siete días más. Se observó además que algunas larvas se movían por toda el área superior de la espuma y de la dieta, no solo dentro y bajo la dieta como era lo usual cuando hubo pocas larvas en la caja. Por esa razón, cuando hubo muchos huevos, se colocaron en cajas de mayor tamaño aumentando la cantidad de dieta, con lo cual mejoró la sincronización en el desarrollo de larvas. Además, se quitó la tapa y se introdujeron en una bolsa o funda hecha de tela tipo Tergal, favoreciendo de ésta manera la aireación y la prevención de contaminación. Bajo este sistema, el desarrollo de las larvas se sincronizó y a los 10 días de colocados los huevos todas las larvas se transformaron a pupas. La falta de nutrientes y aireación pudieron incidir en el comportamiento errático de las larvas y la falta de sincronización del período larval (Taylor 2014) ${ }^{4}$.

\section{Mantenimiento de la cría}

Durante todo el proceso se presentaron problemas de contaminación de la cría, siendo más insidiosos al principio de la actividad. Dichas contaminaciones seguramente afectaron la eficiencia y la productividad del proceso; sin embargo, conforme se mejoró la sincronización en el manejo del sistema y la asepsia de las cajas de cría, se logró el establecimiento de la cría con resultados satisfactorios. Cabe mencionar que conforme se mantenga la cría, se deben realizar cambios que mejoren la productividad y asepsia del sistema. Lo anterior coincide con lo expuesto por Fisher et al. (1999), quienes indican que se debe evitar la contaminación de la colonia para no afectar negativamente la productividad, ya sea por competencia, interferencia, parasitismo, predación o enfermedad. Se ha reportado que algunos ácaros y otros insectos pueden causar problemas por competencia por el sustrato o por afectación directa del insecto. Además; hongos como Penicillium, Aspergillus o Neurospora han aparecido durante el desarrollo de inmaduros principalmente cuando se utilizan jaulas de madera, situación que solventó Rodríguez (2007), empleando jaulas plásticas y recolecta diaria de camas de cría en papel tipo Kraft.

Se encontraron problemas de contaminación en las cajas de cría de adultos, principalmente con hongos de los géneros Aspergillus y Penicillium y con otros insectos que ingresaron a la caja y ovipositaron en el mismo sitio que las moscas del establo. Esto se resolvió cerrando las aberturas (puertas con cinta adhesiva y agujeros en las mallas antiáfidos con silicona). En las cajas de cría de larvas también se identificaron los hongos antes mencionados, acaros y otros insectos de las familias Drosophilae, Mycetophilidae, Sciaridae y Staphlinidae (Taylor 2014) .

En el área de cría, se observaron parasitoides (Figura 8), los cuales se cree venían en los rastrojos de piña traídos al laboratorio. Se

4 Taylor, D. 2014. Cría de la mosca del establo (entrevista). USA-University of Nebraska, USDA-ARS. Comunicación personal.

5 Taylor, D. 2014. Identificación de insectos contaminantes (entrevista). USA-University of Nebraska, USDA-ARS. Comunicación personal. 
identificó el Pteromalidae de $3 \mathrm{~mm}$ de largo del género Spalangia sp (Hanson 2014) .

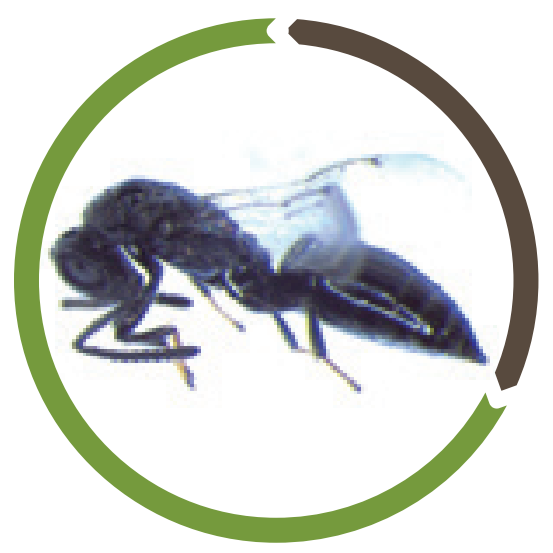

Figura 8. Parasitoide del género Spalangia sp. capturado en el área de cría de larvas y pupas. San José, Costa Rica, 2013.

\section{Pupas}

\section{Limpieza y recolección de pupas}

Las pupas fueron recolectadas en las cajas de desarrollo de larvas. En un inicio al no estar el proceso sincronizado se revisaron todos los días a partir del día siete hasta el día 40 y las encontradas se pasaron a la caja de cría de adultos correspondientes al siguiente ciclo (F1, F2, F3...). Cuando se logró sincronizar el ciclo, las cajas se revisaron los días 10,11 y 12, periodo en el cual salieron todas las pupas descartándose posteriormente los desechos incluyendo las larvas que no habían pasado a pupa.

Las pupas, en su mayoría, se encontraron dentro y entre la espuma y la tela. Las larvas de último estadio, migraron de la dieta y tendieron a formar su estadio de pupa en esa zona, lo que demuestra que este es un mejor micro hábitat que la dieta. Esta situación, facilitó el conteo y extracción de pupas (Figura 9) ya que pocas fueron observadas entre la dieta, y las que quedaron ahí se sacaron por flotación (agregando agua a la caja de cría el día 12).

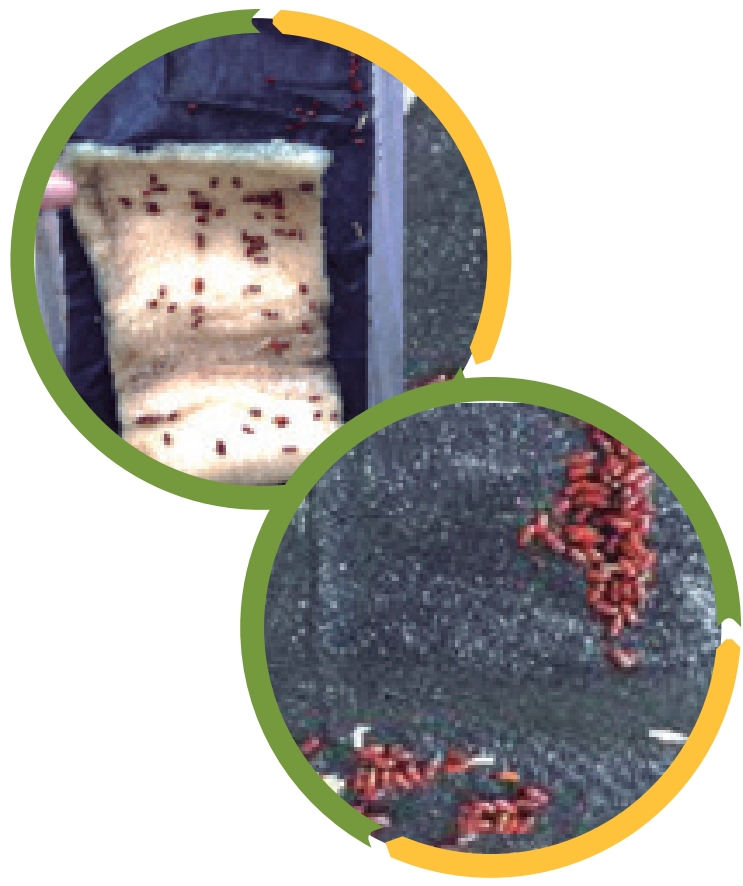

Figura 9. La mayoría de larvas forman las pupas en la tela negra y dentro de la espuma. San José, Costa Rica, 2013.

A fin de obtener otro parámetro para evaluar la calidad de las generaciones desarrolladas en el laboratorio, se midió la longitud de 51 pupas de la Fs, las cuales midieron entre 4,5 y $5,5 \mathrm{~mm}$ (Cuadro 2). Las pupas provenientes de larvas de más de 12 días luego de emergidas en laboratorio tendieron a tener una longitud más pequeña (Figura 10), por lo que las larvas con más de 12 días se descartaron de la cría.

Cuadro 2. Longitud de las pupas Fs. San José, Costa Rica. 2013.

\begin{tabular}{|c|c|}
\hline Estadio & Pupa \\
\hline Parámetro & Longitud, mm \\
\hline Valor promedio & 5,0 \\
\hline Desviación estándar & 0,19 \\
\hline Valor mínimo & 4,5 \\
\hline Valor máximo & 5,5 \\
\hline $\mathrm{N}$ & 51 \\
\hline
\end{tabular}

La longitud de pupas podría utilizarse para evaluar la calidad de las diferentes generaciones y de la adecuada alimentación en cada ciclo, ya que como se había mencionado, las larvas que se desarrollaron en un sustrato nutricionalmente limitado (pinzote) fueron alargadas y delgadas y sus pupas y adultos fueron más pequeños.
6 Hanson, P. 2014. Identificación de insectos contaminantes (entrevista). Universidad de Costa Rica. Comunicación personal. 


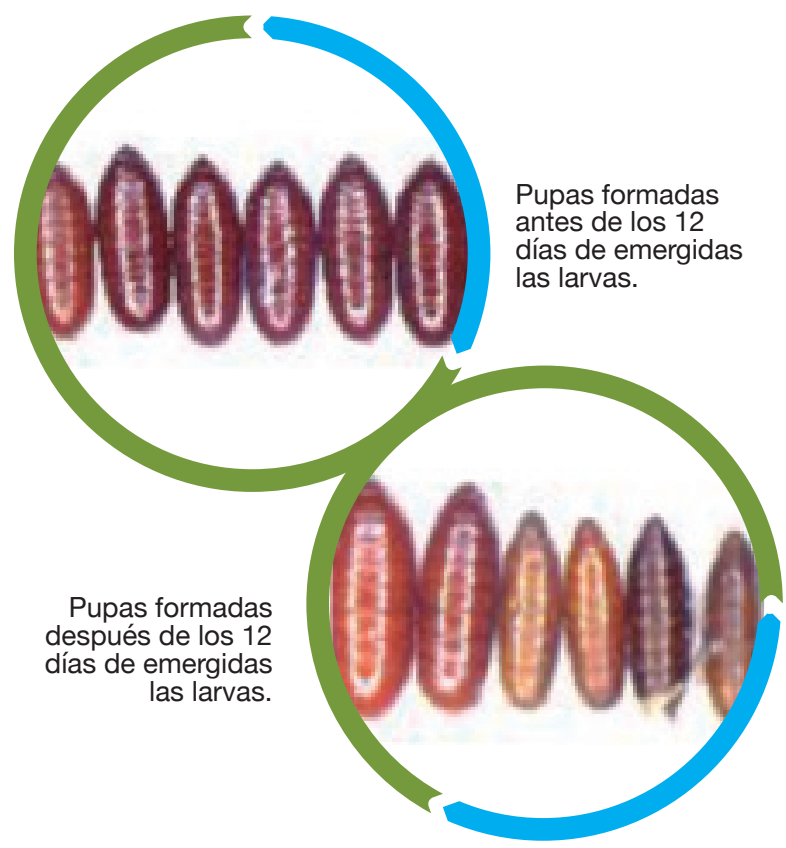

Figura 10. Longitud de las pupas antes y después de los 12 días de emergidas las larvas. San José, Costa Rica, 2013.

Se evaluó la eficiencia de la transformación de pupas a adultos, obteniéndose valores del 60 al $76 \%$ durante los ciclos Fs, F1, F2 y F3. En relación con lo anterior se observaron pupas que contenían la mosca momificada y otras con agujeros pequeños y otros más grandes, quizás producto de los contaminantes encontrados en la cría (parasitoides, ácaros, hongos u otros)

\section{Ciclo Biológico}

En la Figura 11, se observa la duración del ciclo biológico completo de la mosca del establo obtenido bajo condiciones de laboratorio del INTA. El ciclo completo, tomando en cuenta los 10 días en que el adulto tarda en iniciar la oviposición, puede durar de 28 a 32 días según sean las condiciones de temperatura, humedad y nutrición, los adultos permanecieron vivos hasta por 28 días y ovipositaron hasta por 17 días. Foil y Hogsette (1994), indican que las hembras inician la cópula entre los días 3 y 5 después de emerger y colocan huevos dos días después siempre y cuando haya suficientes tomas de sangre para la fecundación de los huevecillos. Salem et al. (2012), lograron mantener la producción de hembras vivas por 24 días, ovipositando por 14 días desde el séptimo día hasta el día 20 con máximo de oviposición entre 11 y 16 días.

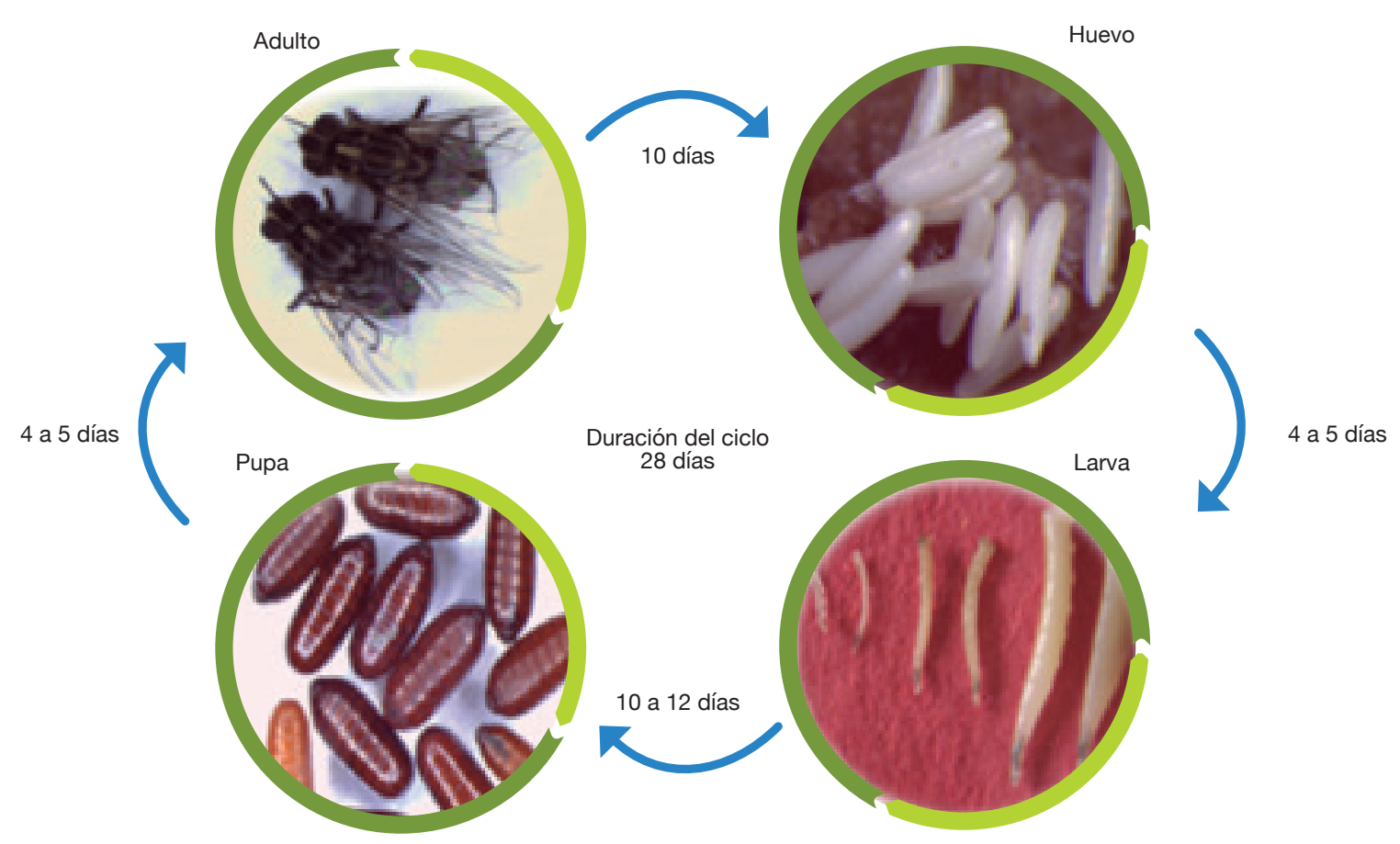

Figura 11. Ciclo de la mosca del establo en condiciones de laboratorio. San José, Costa Rica, 2013. 
En la Figura 12, se muestran los períodos en días de los diferentes estadios de la mosca del establo según la fuente de alimento para larvas; con dieta (lado izquierdo) y con pinzote de plátano (lado derecho).

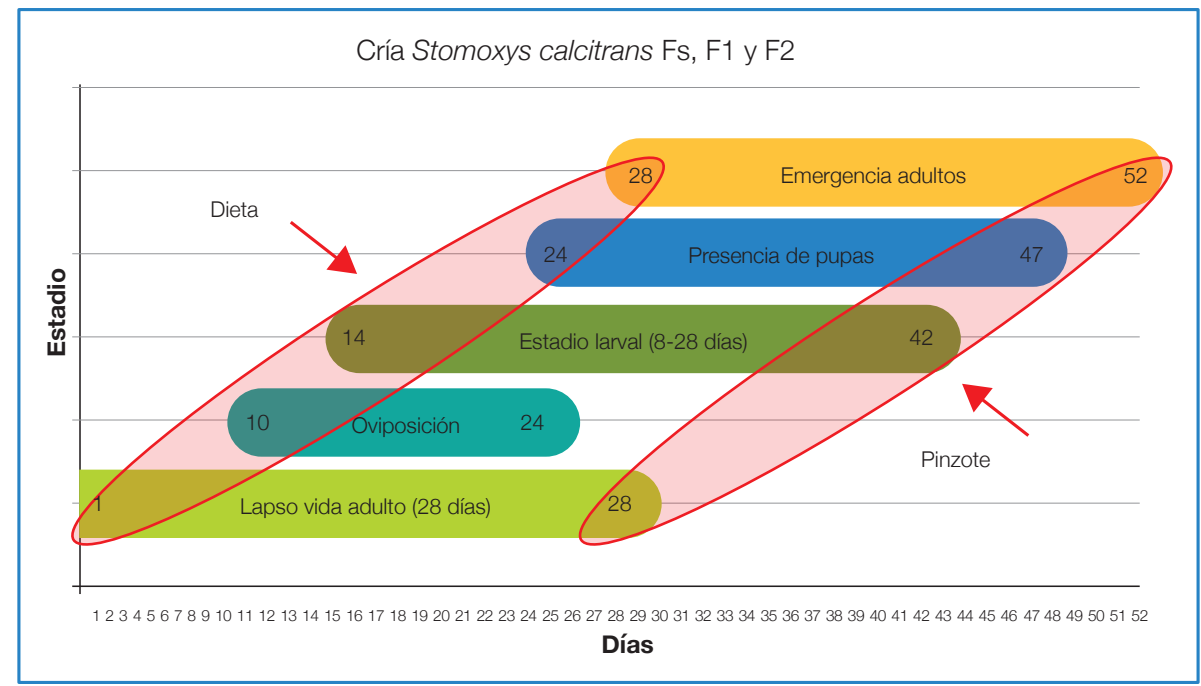

Figura 12. Periodos de los diferentes estadios de la mosca del establo en condiciones de laboratorio y su duración en días, según el tipo de alimentación de la larva. San José, Costa Rica, 2013.

Como se mencionó, el desarrollo de larvas, bajo las condiciones nutricionales a las que fueron expuestas, incidieron en gran medida en el tiempo que transcurrió desde el primer estadio hasta la formación de la pupa. Cuando se utilizó pinzote como medio de desarrollo, el período larval fue el más largo, tardándose hasta 28 días en pasar a pupa, quizás debido a que el pinzote no ofrecía suficientes nutrientes para el paso de un estadio a otro. Por lo contrario, cuando los huevos fueron colocados en una dieta a base de salvado de trigo y harina de pescado, el paso de larva a pupa fue el más rápido (10-12 días). El salvado de trigo contiene carbohidratos, vitaminas, aminoácidos, sales y ácidos grasos y la harina de pescado aporta aminoácidos, sales y otros nutrientes que pudieron favorecer un período larval más corto (Botanical sf y FUNIBER sf).

\section{Calidad de la cría}

Un sistema de cría adecuado es capaz de proporcionar un ambiente donde al menos un $75 \%$ de los huevos resultan viables, los estadios juveniles deben tener un tamaño semejante a los encontrados en campo y los adultos puedan aparearse, poner huevos viables y reproducirse continuamente sin perder vigor o la fecundidad (Singh y Moore 1985).
Según las observaciones y los datos obtenidos se podrían utilizar los siguientes parámetros para evaluar la cría establecida en laboratorio.

- Proporción macho: hembra en una cría establecida: 1:1

- Índices frontales:

- Machos: 0,36 $\pm 0,05$

- Hembras: 0,56 $\pm 0,04$

- Longitud de adultos:

- Machos: 5,50 $\pm 0,50 \mathrm{~mm}$

- Hembras: 5,82 $\pm 0,67 \mathrm{~mm}$

- Longitud de las pupas: 5,04 $\pm 0,19 \mathrm{~mm}$

- Porcentaje de emergencia de adultos: mayor al $60 \%$

- Días de emergencia de adultos luego de formada la pupa: 4-5

- Día de emergencia de larvas luego de la oviposición: 4-5

- Días de formación a pupas: 10-12

- Larvas sobre la dieta o la tela negra: No presentes (las larvas deben permanecer dentro y bajo la dieta) 


\section{Consideraciones finales}

- No utilizar cajas de vidrio para la cría.

- Utilizar cajas de plástico para el mantenimiento de adultos o bien cajas de madera con malla antiáfidos previamente lavadas con jabón, cloro y suficiente agua. Se podrían evaluar las cajas de aluminio.

- Revisar diariamente las cajas de cría de adultos y larvas y cerrar cualquier abertura que permita la entrada de otros insectos que contaminan la cría.

- Colocar malla antiáfidos en las ventanas del cuarto de cría a fin de evitar el ingreso de otros insectos que afecten el proceso.
- Utilizar sangre fresca para la alimentación. No se debe utilizar sangre de más de 2 semanas en refrigeración.

- En caso de que se deteriore la calidad de la cría, se podría evaluar la introducción de moscas silvestres cada tres ciclos en laboratorio, a fin de mantener la calidad genética de la cría.

- Se observó en los diferentes ciclos, que los primeros adultos en emerger son machos en su mayoría. Esta observación debe ser validada.

- Evaluar otras fuentes nutricionales a las indicadas en este estudio.

- Evaluar la viabilidad de pupas a diferentes temperaturas de refrigeración y congelación.

\section{AGRADECIMIENTO}

A los médicos veterinarios y técnicos del Servicio Nacional de Salud Animal (SENASA) que laboran en el matadero El Arreo en San Antonio de Belén, los cuales sangraron regularmente el ganado y facilitaron la sangre para el estudio. A los doctores David Taylor y Paul Hanson por las identificaciones de los insectos contaminantes encontrados en la cría.

\section{LITERATURA CITADA}

Ashrafi, S. 1964. The cultivation and nutritional requirements of Stomoxys calcitrans. Bulletin of the world Health Organization. 31(4):519-520.

Bakri G 1959. Massenzucht der Stechfliege, Stomoxys calcitrans $(\mathrm{L})$ in Laboratorium-S. angew. Zool. 46:491-494.

Berkebile, D; Thomas, G; Campell, J. 1994. Overwintering of stable flies (Diptera: Muscidae) in Southeastern Nebraska. Journal of Economic Entomology. 87(6):1555-1563.

Berkebile, A; Weinhold, P; Taylor, D. 2009. A new method for collecting clean stable fly (Diptera: Muscidae) pupae of known age Southwestern Entomologist. 34(4):469-476.
Botanical. sf. Propiedades del salvado de trigo (en línea). Barcelona, España. Consultado el 2 de mayo del 2014. Disponible en http://www.botanical-online.com/salvadodetrigo.htm.

Campau, E; Baker G; Morrison, F. 1953. Rearing stable fly for laboratory tests. Journal of economic entomology. 46:524.

Campbell, J; Skoda, S; Berkebile, D; Boxler, R; Thomas, D; Adams, D; Davis, R. 2001. Effects of stable flies (Diptera: Muscidae) on weight gain of grazing yearling cattle. Journal of Economic Entomology. 94(3):780-783.

Champlain, R; Fisk, F; Dowdy, A. 1954. Some improvements in rearing stable flies. Journal of Economic Entomology. 47(5):940-941. 
Dos Pinos. sf. Leches en Polvo (en línea). Consultado el 3 de mayo del 2014. Disponible en http://www.dospinos.com/app/cms/www/index .php?parent_pk_categoria $=1 \&$ pk_categoria $=20 \& i d$ _menu=133.

Doty, AE. 1937. Convenient method of rearing the stable fly. Journal of Economic Entomology 30(2):367-369.

Fisher, T; Bellows, T; Caltagirone, L; Dahlsten, D; Huffaker, C; Gordh, G. 1999. Handbook of biological control: principles and aplications of biological control. Academic Press. p:25-197.

FUNIBER (Fundación Universitaria Iberoamericana). Sf. Composición nutricional. Harina de pescado (en línea). Consultado el 2 de mayo del 2014. Disponible en http://composicionnutricional.com/ alimentos/HARINA-DE-PESCADO-5

Foil, L; Hogsette, J. 1994. Biology and control of tabanids, stable flies and horn flies. Revue scientifique et technique. International office of epizootics. 13(4):1125-1158.

Gilles, J; David, J; Duvallet, G. 2005. Effects of temperature on the rate of increase of Stomoxys calcitrans and Stomoxys niger niger (Diptera: Muscidae) from La Reunion Island. Journal of Medical Entomology. 42(6):959-965.

Gilles, J; David, J; Duvallet, G; De la Rocque, S; Tillard, E. 2007. Efficiency of traps for Stomoxys calcitrans and Stomoxys niger niger on Reunion Island. Medical and Veterinary Entomology. 21(1):65-69.

Gilles, J; David, J; Lecomte, P; Tillard, E. 2008. Relationships between chemical properties of larval media and development of two Stomoxys species (Diptera: Muscidae) from Reunion Island. Environmental Entomology. 37(1):45-50.

Hargett, L; Goulding, R. 1962. Rearing the Horn fly, Haematobia irritans. Journal Economic Entomology. 55(4): 565-566.

Harwood, R; James, M. 1979. Entomology and human animal health. McMillan. New York. 548 p.
Herrera, M; Montes-Pico, L; Hernández, R. 1994. Abundancia relativa de Stomoxys calcitrans (L) (Diptera: Muscidae) en seis localidades del Pacífico Sur de Costa Rica. Biología Tropical. 39(2):309-310.

Justin, T. 2008. Management and characterization of stable fly larval habitats at round hay bale feeding sites in Pastures. 2008. Tesis PhD. Kansas State University. 109 p.

Martínez, M; Lumaret, J. 2006. Las prácticas agropecuarias y sus consecuencias en la entomofauna y el entorno ambiental. Folia Entomológica Mexicana. 45(1):57-68.

Masmeatathip, R; Ketavan, C; Duvallet, G. 2006. Morphological studies of Stomoxys spp. (Diptera: Muscidae) in central Thailand. Kasetsart Journal. Natural Sciences. 40:872-881.

Parr, H. 1959. Studies on Stomoxys calcitrans $(L)$ in Uganda, East Africa. I. Method of rearing large numbers of Stomoxys calcitrans. Bulletin. Entomology Research. 50(1):165-169.

Rodríguez, A P. 2007. Stomoxys calcitrans: Establecimiento de colonia e efecito de Metarhizium anisopliae sobre seus estágios imaturos. Tesis Mestre enm Ciencias, Instituto de Veterinaria Universidade Federal Rural do Rio de Janeiro. $52 \mathrm{p}$.

Salem, A. 2012. Stomoxys calcitrans (L): morphologie, biologie, rôle vecteur et moyens de lute. Thése Doctorat. Universidad Tolouse. Francia. 149 p.

Salem, A; Franc, M; Jaquiet, M; Bouhsira, E; Lienard, E. 2012. Parasite. Feedinf and breeding aspects of Stomoxys calcitrans (Diptera: Muscidae) under laboratory conditions. Parasite. 19(4):309-317.

Schmidt, C; Harris, R; Hoffman, R. 1967. Mass rearing of the horn fly, Haematobia irritans (Diptera: Muscidae) in the laboratory. Annals of the Entomological Society of America. 60(3):508-510.

Schmidt, C; Harris, R; Hoffman, R. 1968. New techniques for rearing horn flies at Kerrville. Annals of the Entomological Society of America 61(4):1045-1046. 
Scholl, P. 1986. Field populations of Stomoxys calcitrans (L) in Eastern Nebraska. Southwest Entomology. 11(3):155-160.

Singh, P; Moore, R. 1985. Handbook of insect rearing. Vol II. Elsevier Science. p. 153-155.

Solórzano, J; Tevino, J; Hidalgo, E; Gómez, Y; Blanco, H; Apuy, M; González, L; Meneses, D. 2013. Recomendaciones para el manejo de la mosca del establo Stomoxys calcitrans en el cultivo de la piña. San José, Costa Rica, MAG. 32 p.
Taylor, D; Berkebile, D. 2006. Comparative efficiency of six stable fly (Diptera: Muscidae) Traps. Faculty publications. Entomology Department. University of Nebraska. Lincoln Nebraska. Journal of Economic Entomology. 90(4):1414-1419.

Zumpt, F. 1973. The Stomoxyne biting flies of the Word (Diptera: Muscidae). Taxonomy, biology, economic importance and control measures. Stuttgart, Gustav Fisher Verlag 175 p. 
